\title{
Severe Bronchial Asthma in the era of biological treatments
}

\author{
Eleni Manoloudi, Paschalis Steiropoulos \\ Department of Pneumonology, Democritus University of Thrace, Greece
}

Correspondence: Paschalis Steiropoulos MD, PhD, FCCP, Department of Pneumonology, Medical School, Democritus University of Thrace, Dragana 68100,Alexandroupolis, Greece, Tel +30 2551077377, Email steiropoulos@yahoo.com

Received: March 07, 2018 | Published: November 13, 2018

Copyright@ 2018 Manoloudi et al. This is an open access article distributed under the terms of the Creative Commons Attribution License, which permits unrestricted use, distribution, and reproduction in any medium, provided the original author and source are credited.

\begin{abstract}
Severe bronchial asthma is a chronic heterogeneous disease that requires a combination of therapies in order to be sufficiently controlled. A significant number of patients, however, do not achieve adequate control, leading to frequent exacerbations, impaired quality of life and increased health care costs. In recent years, several biological agents for severe asthma treatment have been introduced in the market or are under development. Although, biological treatment regimens for severe asthma are increasing our perspective about future asthma approach for specific asthma phenotypes and endotypes, still certain issues are raised concerning their appropriate use in line with everyday clinical practice.
\end{abstract}

\section{Introduction}

Bronchial Asthma (BA) is a chronic inflammatory disease characterized by bronchial hyper responsiveness (BHR) and reversible airway obstruction, clinically presented with respiratory symptoms such as shortness of breath, wheezing, cough and chest tightness. ${ }^{1}$ Especially severe asthma is heterogeneous with respect to clinical characteristics, physiological measures and biomarker expression (different phenotypes). According to ATS/ERS guidelines, severe asthma requires high doses of inhaled corticosteroids (ICS) plus a second controller (long acting $\beta_{2}$ agonists), and/or systemic corticosteroids to maintain control or remains uncontrolled despite this treatment. ${ }^{2}$ Patients suffering from severe asthma comprise $5-10 \%$ of all asthma patients. ${ }^{3}$ However, severe asthma counts for millions of physician office visits annually, millions of emergency room visits and thousands of hospital discharges. Hence, the financial costs for severe asthma are more than double compared to those for controlled asthma. ${ }^{4}$ In a global basis, billions are spent annually on severe asthma care and the associated costs exceed those of TB and HIV combined. ${ }^{5}$ Due to these unmet needs, novel therapeutics have been developed, whereas individual approaches are urgently required.

In this brief editorial the available treatment options for severe asthma, focusing on biological therapies, their mechanisms of action and the issues raised concerning their optimum use would be discussed.

\section{Conventional therapies for severe bronchial asthma}

Asthma treatment aims at controlling symptoms, reducing exacerbations and improving lung function in an effective and longlasting manner. ${ }^{6}$ Standard treatment for severe asthma includes high doses of ICS which constitute the therapeutic asthma basis, control symptoms, reduce airway inflammation, and minimize future risks such as exacerbations, and $\beta_{2}$ agonists that relieve breakthrough symptoms. ${ }^{7}$ Other options are cysteinyl leukotriene receptor antagonists (controllers) and theophylline (controller). ${ }^{7}$ Despite these therapies, many patients do not achieve adequate disease control. Therefore, add-on treatment is considered, which usually refers to a biological agent, leaving oral corticosteroids (OCS) as the last option due to the number of adverse effects. Major factors that impede successful asthma management are asthma heterogeneity and variability to treatment response. ${ }^{6}$ Therefore, the identification of different asthma phenotypes/endotypes could lead to better treatment approaches. ${ }^{6,7}$

\section{Biological therapies for severe bronchial asthma}

Aiming at better asthma management and personalized treatment, novel biological therapies have been recently developed. The main advantage of those therapies is that they target specific molecular pathways of asthma and thus, they are more efficient and reduce nonspecific adverse effects of traditional therapies. ${ }^{6}$

There are two prevailing asthma endotypes based on T helper 2 and innate lymphoid cell activity and mediators, i.e. T2-high and T2low asthma endotype. ${ }^{8}$ Treatment options for T2-low asthma are restricted. ${ }^{8}$ On the other hand, the majority of biological regimens, which are currently approved or being under development, aim at the type 2-high asthma endotype, characterized by a prevailing role of type 2 cytokines IL-4, IL-5 and IL-13, and IgE. ${ }^{9}$ Also, there are other under development biological agents that target thymic stromal lymphopoietin, prostaglandin D2 receptor, IL-25 and IL-33. 


\section{Anti-immunoglobulin E}

More than $50 \%$ of patients with poorly controlled asthma have allergic immunoglobulin E (IgE)-mediated asthma. ${ }^{10} \mathrm{IgE}$ has a crucial upstream role in the inflammatory cascade of allergy and allergic asthma. ${ }^{6}$ Omalizumab is the only biological anti- $\operatorname{IgE}$ agent currently approved for humans. ${ }^{10}$ It was approved by the FDA in 2003 and by the European Union in 2005 as add-on treatment for patients aged $>12$ years with severe persistent allergic asthma who have serum total $\operatorname{IgE}$ levels $30-700 \mathrm{IU} / \mathrm{mL} .{ }^{10}$

It is a recombinant DNA derived humanized IgG1 monoclonal antibody, which was originally constructed as a murine antibody selectively binding to human IgE. ${ }^{11}$ It has two main mechanisms of action: 1) it binds exclusively to circulating $\operatorname{IgE}$ in the blood and interstitial space and promotes its depletion and 2) it inhibits $\operatorname{IgE}$ binding to high-affinity (FcoRI) or low-affinity receptors (FcoRII) on basophils, mast cells and dendritic cells. ${ }^{10}$ As a result, it hinders the release of inflammatory mediators from mast cells reducing the recruitment of inflammatory cells, especially eosinophils, into the airways. ${ }^{11}$

As reported in clinical trials, omalizumab contributes to reduction in exacerbation rates, fewer emergency department visits and asthma related hospitalizations, better asthma symptom control, reduction in ICS or oral CS dose and improvement in asthma-related quality of life $(\mathrm{QoL}){ }^{6}$

\section{Anti-interleukin 5}

Since 1990, Bousquet et al. ${ }^{12}$ described the association between eosinophilia and asthma severity. Although, eosinophils as multifunctional leukocytes play a protective role against invading pathogens such as virus and bacteria, they are also involved in the pathogenesis of allergic diseases, including allergic asthma. ${ }^{13}$ Thus, it is estimated that about $20 \%$ of patients with refractory asthma demonstrate eosinophilic inflammation. ${ }^{13}$

In allergic asthma, eosinophils accumulate and release granule proteins, reactive oxygen species, lipid mediators and cysteinyl leukotrienes that can induce cellular damage, airway hyper responsiveness and mucus hyper secretion. ${ }^{14,15}$

IL-5, produced by $\mathrm{T}$ helper 2 cells, is the leading cytokine that is engaged in eosinophil production, maturation, activation, accumulation, survival and suppression of eosinophil apoptosis at the site of inflammation. ${ }^{14}$ In humans IL-5 receptor (IL-5R) is expressed exclusively on eosinophils and basophils. ${ }^{15}$

Mepolizumab was the first therapeutic mAb (humanized IgG1, Kappa, mAb) against human IL-5 that was approved by the FDA in 2015, as add-on maintenance treatment for patients with severe asthma aged $>12$ years, and with eosinophilic phenotype. ${ }^{16}$ It binds free IL-5 with high affinity and prevents it from binding to IL-5Ra receptor, which is expressed on eosinophils. ${ }^{14}$ In this manner, it deters IL-5 signaling and over-expressing functions of eosinophils, including release of chemicals that lead to tissue damage. . $^{13,14}$

In clinical trials, mepolizumab has been shown to decrease asthma exacerbations and enhance lung function and asthma-related QoL. Moreover, it was found to deplete blood and sputum eosinophil counts, and was generally well tolerated. ${ }^{10}$
In 2016, FDA approved reslizumab as add-on maintenance treatment for severe eosinophilic asthma in patients $>18$ years. ${ }^{17}$ Reslizumab is also a humanized monoclonal antibody against IL-5. The mechanism in which reslizumab acts is the same as mepolizumab. Data from BREATH studies demonstrated that Reslizumab reduces the likelihood of exacerbation and improves asthma control, lung function and health-related (HR) QoL in patients with inadequately controlled eosinophilic asthma. ${ }^{18}$ Furthermore, it reduces blood and sputum eosinophils and is generally well tolerated. Regarding the adverse events (AE), anaphylaxis and malignancies have been rarely reported. ${ }^{18}$

However, long-term clinical trials and further real life experience is necessary in order to establish its clinical benefit durability. ${ }^{18}$ Also, there is lack of studies comparing directly Reslizumab with Mepolizumab. ${ }^{18}$

Benralizumab (MEDI-563) has recently received FDA approval (on 14 November 2017) for add-on maintenance treatment of patients aged $>12$ years, with severe asthma and with eosinophilic phenotype. ${ }^{19}$ MEDI-563 is a mAb (glycoengineered non-fucosylated humanized IgG1, Kappa, mAb) that binds to human IL-5Ra, which is expressed only in eosinophils and basophils, and thus it inhibits IL-5 signaling. ${ }^{13}$ In addition, the $\mathrm{Fc}$ region of benralizumab binds to $\mathrm{Fc} \gamma$ receptor on natural killer cells and macrophages, and induces secretion of cytotoxic mediators, such as perforin, which promote eosinophil death through antibody dependent cellular cytotoxicity (ADCC). ${ }^{13}$

The clinical trials conducted so far documented direct, rapid and near-complete depletion of eosinophils within 24 hours, leading to significant reduction in annual exacerbation rates, development in lung function and asthma score, especially for patients presenting with blood eosinophil levels $\geq 400$ cells/ $\mu$ l. Moreover, no adverse events have been observed. ${ }^{14,19}$ At present, ongoing clinical trials aim at further drug assessment, ${ }^{20}$ anticipating also the EU approval. Benralizumab remains to be proven more efficient in the treatment of asthma through ADCC depletion of eosinophils and basophils rather than through passive removal of IL-5. ${ }^{14}$

\section{Anti-interleukin 13 and anti-interleukin 4}

IL-13 and IL-4 are involved in IgE synthesis, eosinophil recruitment, mucus secretion and airway remodeling. IL-13 has a critical role in the development of airway hyper responsiveness and acts through the low-affinity IL-13 receptor a1 (IL-13Ra1) and IL-4Ra complexes (IL13Ra1/IL-4Ra complex). IL-4 shares IL-4Ra with IL-13. ${ }^{21}$

Lebrikizumab was the first IgG4 humanized mAb targeting IL13 , binding soluble IL-13 and inhibiting its link to the receptor. ${ }^{21}$ Hence, it blocks the signaling pathway through IL-4R $\alpha / \mathrm{IL}-13 \mathrm{R} \alpha 1 .^{21}$ Nevertheless, its clinical trial in asthma was recently terminated not achieving the primary endpoint (significant reduction in the rate of asthma exacerbations over 52 weeks). ${ }^{21}$

Tralokinumab is another IgG4 mAb to IL-13 that blocks binding and signaling of IL-13 to IL-13 receptors. Tralokinumab also failed to achieve the primary endpoints of two Phase III trials; more specifically, it did not result in a statistically-significant reduction of the annual asthma exacerbation rate in patients with severe, uncontrolled asthma and elevated levels of a biomarker, Fractional exhaled Nitric Oxide $(\mathrm{FeNO}){ }^{22}$ 
Dupilumab is a fully human $\mathrm{mAb}$ directed against the IL-4Ra subunit of IL-4 and IL-13 receptors. It blocks the IL-4Ra receptor and inhibits signaling of both IL-4 and IL-13. Dupilumab is currently under clinical development and its efficacy as add-on treatment in patients with uncontrolled persistent asthma is still being evaluated. So far, it has shown promising results, including decrease in the frequency of severe exacerbations, significant improvement of asthma symptoms, of lung function and of asthma related QoL. ${ }^{6}$

\section{Tumour Necrosis Factor- $\alpha$ (TNF- $\alpha$ )}

It has been observed that TNF- $\alpha$ levels are increased in bronchial biopsies and induced sputum from subjects with severe asthma. ${ }^{23}$ ICS fail to reduce TNF- $\alpha$ levels in airways and this could explain their limited effects in severe asthma. ${ }^{24}$ Hence, it is likely that treatments blocking TNF- $\alpha$ could contribute to better asthma management. ${ }^{24}$ TNF- $\alpha$ is released from mast cells and macrophages in response to immunological stimulation. In the airways, TNF- $\alpha$ evokes inflammatory responses mainly through increased release of proinflammatory and chemotactic mediators and upregulation of adhesion molecules. These events cause chronic eosinophilic and neutrophilic infiltration and irreversible airway remodeling. TNF-a produces its pleotropic effects by interacting with two receptor subtypes, p55 and p75. In addition, TNF- $\alpha$ directly affects the airway smooth muscles leading to increased airway hyper-responsiveness..$^{23,24}$

Etanercept (ETN) is a genetically engineered recombinant protein which consists of two molecules of the extracellular portion of p75 TNF receptor, fused to the Fc portion of a human IgG1. ${ }^{23}$ ETN binds TNF- $\alpha$ and lymphotoxin- $\alpha$ with high affinity ${ }^{23}$ Etanercept is mainly directed towards airway smooth muscle. ${ }^{24}$ Although in clinical trials ETN was well tolerated, clinical efficacy was not shown in target population. ${ }^{23}$ Consequently, long-term studies in specific subsets of asthma patients may be needed to adequately assess the clinical efficacy of ETN in this population. ${ }^{23}$

\section{Therapies for type 2-low asthma}

Importantly, type 2-low asthma patients respond poorly either to corticosteroids or to currently approved biological agents. ${ }^{6}$ Therefore, emerging investigational approaches target the IL-17 receptor. ${ }^{6}$ IL17 is involved in proinflammatory and allergic responses. It induces the production of many cytokines (including TNF- $\alpha$ ) and chemokines, which can also cause airway remodeling. ${ }^{25}$ However, brodalumab, an anti-IL-17 agent, was not shown to be efficient. ${ }^{6}$ Hence, treatment options for type 2-low asthma remain an unmet need. ${ }^{6}$

\section{Where do we stand?}

Outcomes from anti-IL-5 clinical trials (Benralizumab, Mepolizumab) confirm the complexity of eosinophil biology, as they have shown that despite of low post-treatment circulating eosinophil levels, eosinophils continue to be present in lung tissue. ${ }^{14}$ This indicates an alternative mechanism to IL-5 for eosinophil initiation, recruitment, activation and survival in the tissues. ${ }^{14}$ Maybe a combination therapy with anti-IL-4/13 and anti-IL-5 mAbs could synergistically have better results in restriction of tissue eosinophilia. ${ }^{14}$ Furthermore, there are other equally important cytokines to IL-5 for eosinophil survival, such as IL-3 and GM-CSF, that could explain the eosinophil persistence during IL-5 blockage, and also act as potential targets for novel drugs. ${ }^{15}$
Given the growing availability of biological agents for severe asthma, it is necessary to determine which patients will benefit the most from each therapy and which biomarkers are the most specific and practical to identify these patients. ${ }^{18}$ Hence, further research, especially headto-head trials, is required to determine specific populations. ${ }^{6}$ In addition to trials and taking into consideration the heterogeneity of severe asthma and inter individual variability in treatment response, research for appropriate biomarkers needs to be continued, as they could help to define asthma phenotypes and endotypes. ${ }^{6}$ This would be also especially useful in childhood asthma, as this is the right time for potential prevention strategies. ${ }^{6}$ All these will enable successful asthma management and real application of personalized medicine. ${ }^{6}$ Also, because of the high cost of these therapies, the identification of likely responders will result in cost effectiveness.

Finally, in the absence of head-to-head clinical trials, it is difficult to decide which agent to select; so the choice could depend on the currently available efficacy data, the route of administration (intravenous or subcutaneous) and the patient age. ${ }^{18}$ Therefore, care pathways would be helpful in elucidating the first choice of treatment for severe asthma patients, especially for new cases, when to switch from one $\mathrm{mAb}$ to another and when to consider stopping them, due to their high cost. ${ }^{6}$

\section{Conclusion}

The emerging biological treatments show promising results and provide hope for successful management of severe asthma. However, efforts for improved disease understanding are constant. The identification of disease endotypes and appropriate biomarkers is in progress. This biology-based approach hopefully will lead in accurate diagnosis and determination of the best treatment for each patient subgroup, leading to the era of personalized treatment and thus, successful asthma control and more efficient use of healthcare resources. All these findings indicate that in the future there will be more than one treatment options for each patient and further research is needed in order to set the criteria for categorizing every severe asthma patient.

\section{Conflicts of interest}

The authors declare no conflict of interest related to the context of the manuscript.

\section{References}

1. Karo-Atar D, Munitz A. Is Asthma Paying the Toll? Am J Respir Cell Mol Biol. 2018;58(1):3-4.

2. Chung KF, Wenzel SE, Brozek JL, et al. International ERS/ATS guidelines on definition, evaluation and treatment of severe asthma. Eur Respir J. 2014;43(2):343-373.

3. Busse WW, Banks-Schlengel S, Wenzel SE. Pathophysiology of severe asthma. J Allergy Clin Immunol. 2000;106:1033-1042.

4. Sullivan SD, Rasouliyan L, Russo PA, et al. Extent, patterns, and burden of uncontrolled disease in severe or difficult-to-treat asthma. Allergy. 2007;62(2): 126-133.

5. World Health Organization. Bronchial asthma. Accessed March 2018, at http://www.who.int/mediacentre/factsheets/fs206/en/

6. Humbert M, Busse W, Hanania NA. Controversies and oppotunities in severe asthma. Wolters Kluwer Health. 2018;24:83-93. 
7. Global Initiative for Asthma (GINA). Global strategy for asthma management and prevention. Accessed March 2018, at http://ginasthma. org/2018-gina-report-global-strategy-for-asthma-management-andprevention/

8. Pepper AN, Renz H, Casale TB, et al. Biologic Therapy and Nove Molecular Targets of Severe Asthma. J Allergy Clin Immunol Pract. 2017;5(4): 909-916

9. Bousquet J, Brusselle G, Buhl R, et al. Care pathways for the selection of a biologic in severe asthma. Eur Respir J. 2017;50(6):1701782.

10. Papathanasiou E, Loukides S, Bakakos P. Severe asthma: anti-IgE or antiIL-5? Eur Clin Respir J. 2016;3:31813.

11. Presta LG, Lahr SJ, Shields RL, et al. Humanization of an antibody directed against IgE. J Immunol. 1993;151(5): 2623-2632.

12. Bousquet J, Chanez P, Lacoste JY, et al. Eosinophilic inflammation in asthma. N Engl J Med. 1990;323(15):1033-1039.

13. Yanagibashi T, Satoh M, Nagai Y, et al. Allergic diseases: From bench to clinic - Contribution of the discovery of interleukin-5. Cytokine. 2017;98:59-70.

14. Mukherjee M, Sehmi R, Nair P. Anti-IL5 therapy for asthma and beyond. World Allergy Organ J. 2014;7(1):32.

15. Kolbeck R, Kozhich A, Koike M, et al. MEDI-563, a humanized antiIL-5 receptor a mAb with enhanced antibody-dependent cell-mediated cytotoxicity function. J Allergy Clin Immunol. 2010;125(6):1344-1353.

16. Federal Drug Administration. Accessed January 2018, at https://www. accessdata.fda.gov/drugsatfda_docs/label/2015/125526Orig1s000Lbl. pdf
17. Federal Drug Administration. FDA approves Cinqair to treat severe asthma. 2016. Accessed January 2018, at https://www.fda.gov/ NewsEvents/Newsroom/PressAnnouncements/ucm491980.htm

18. Deeks ED, Brusselle G. Reslizumab in Eosinophilic Asthma: A Review. Drugs. 2017:77(7):777-784.

19. Fasenra (benralizumab) receives US FDA approval for severe eosinophilic asthma. 2017. Accessed January 2018, https://www.astrazeneca.com/ media-centre/press-releases/2017/fasenra-benralizumab-receives-us-fdaapproval-for-severe-uncontrolled-eosinophilic-asthma-1411201

20. ClinicalTrials.gov. Accessed January 2018, at https://www.clinicaltrials. gov/ct $2 /$ results?term=benralizumab $\&$ Search=Search

21. Özdemir SK, Bavbek S. Prospects for new and emerging therapeutics in severe asthma: the role of biologics. Expert Rev Respir Med. 2017: 11(6):505-512.

22. Astra Zeneca. Astra Zeneca provides update on tralokinumab Phase II programme in severe, uncontrolled asthma. (2017) Accessed January 2018, at https://www.astrazeneca.com/media-centre/press-releases/2017/ astrazeneca-provides-update-on-tralokinumab-phase-iii-programme-insevere-uncontrolled-asthma-01112017.html

23. Holgate ST, Noonan M, Busse CW, et al. Efficacy and safety of etanercept in moderate-to-severe asthma: a randomised, controlled trial. Eur Respir J. 2011;37(6):1352-1359.

24. Holgate ST, Polosa R. The mechanisms, diagnosis, and management of severe asthma in adults. Lancet. 2006;368(9537):780-793.

25. Jin W, Dong C. IL-17 cytokines in immunity and inflammation. Emerg Microbes Infect. 2013;2(9):e60. 